Chirurg 2019 $\cdot 90: 239$

https://doi.org/10.1007/s00104-018-0777-0

Online publiziert: 4. Januar 2019

(c) Springer Medizin Verlag $\mathrm{GmbH}$, ein Teil von Springer Nature 2018

\section{CrossMark}

\section{Originalpublikation}

Wang B-Y, Wu S-C, Chen H-C et al (2018)

Survival after neoadjuvant

chemoradiotherapy and oesophagectomy

versus definitive chemoradiotherapy for

patients with oesophageal squamous cell carcinoma. Br J Surg. https://doi.org/10.1002/ bjs. 11004

Hintergrund. Das Ösophaguskarzinom ist die sechsthäufigste tumorbedingte Todesursache weltweit. Obwohl in Nordamerika und Westeuropa Adenokarzinome (AC) überwiegen, ist das Plattenepithelkarzinom (SCC) wesentlich häufiger. Sowohl die neoadjuvante Radiochemotherapie mit Resektion $(\mathrm{CRT}+\mathrm{R})$ als auch die definitive Radiochemotherapie (dCRT) stellen beim lokal fortgeschrittenen SCC etablierte Therapiekonzepte dar. Die beiden Therapiekonzepte wurden im Rahmen dieser retrospektiven Arbeit verglichen.

Methoden. Für diese Registerstudie aus Taiwan wurden SCC-Patienten im lokal fortgeschrittenen Stadium (UICC II/III) eingeschlossen, die im Zeitraum 2008 bis 2014 entweder eine dCRT oder eine CRT+R erhielten.

Ergebnisse. Insgesamt 4078 Patienten mit lokal fortgeschrittenem SCC erhielten eine dCRT, 1754 Patienten eine $\mathrm{CRT}+\mathrm{R}$. In der multivariaten Analyse stellte die Therapiemodalität einen unabhängigen prognostischen Faktor dar. Patienten in der CRT+R-Gruppe hat-

M. Schrempf · M. Anthuber ${ }^{1}$

${ }^{1}$ Klinik für Allgemein-, Viszeral-Transplantationschirurgie, Klinikum Augsburg, Augsburg, Deutschland

\title{
Definitive Radiochemotherapie vs. neoadjuvante Radiochemo- therapie und Resektion beim Plattenepithelkarzinom des Ösophagus
}

ten im Vergleich zu Patienten in der dCRT-Gruppe ein signifikant geringeres Sterberisiko (Harzard Ratio [HR] = 0,45; $95 \%$-Konfidenzintervall [KI] 0,40-0,51; $p<0,001)$. Mittels "propensity-score matching" wurden zwei gleiche große Gruppen à 1661 Patienten gebildet. Das 3-Jahres-Gesamtüberleben (OS) unterschied sich hier mit 17,9\% in der dCRTGruppe und $41,1 \%$ in der CRT+R-Gruppe signifikant $(p<0,001)$. Die $C R T+R$ war auch bei der getrennten Betrachtung von Patienten im Stadium UICC II und UICC III mit einem signifikant besseren Gesamtüberleben assoziiert.

Diskussion und Fazit. In dieser Arbeit zeigte sich für die CRT+R-Gruppe im Vergleich zur dCRT-Gruppe ein deutlicher Überlebensvorteil. Die Ergebnisse stehen damit im Widerspruch zu den Langzeitergebnissen der relativ alten randomisierten Studie von Stahl et al. und der FFCD9102-Studie von Bedenne et al., die keine signifikanten Unterschiede in Bezug auf das Gesamtüberleben nachweisen konnten [1, 4]. Allerdings steht insbesondere die letztgenannte Arbeit aufgrund ihres mangelhaften Designs und methodischer Mängel in der Kritik.

In der vorliegenden Arbeit muss trotz eines "propensity-score matching" von einem erheblichen Bias ausgegangen werden. Da für einen großen Teil der Patienten keine Informationen zu Grading und Tumorlokalisation vorliegen, wird die Interpretation weiter erschwert.
Allerdings werden die Ergebnisse durch zwei Arbeiten aus den USA bestätigt, die sowohl für das SCC als auch für das AC einen signifikanten Überlebensvorteil für die $C R T+R$ nachweisen konnten [2, 3]. Die Frage nach dem besten Therapiekonzept beim SCC ist daher bislang nicht ausreichend beantwortet. Dies muss mit dem Patienten im Rahmen der gemeinsamen Entscheidungsfindung erörtet werden.

\section{Korrespondenzadresse}

Prof. Dr. M. Anthuber

Klinik für Allgemein-, Viszeral-Transplantationschirurgie, Klinikum Augsburg

Stenglinstr. 2, 86156 Augsburg, Deutschland matthias.anthuber@klinikum-augsburg.de

Interessenkonflikt. M. Schrempf und M. Anthuber geben an, dass kein Interessenkonflikt besteht.

\section{Literatur}

1. Bedenne L, Michel P, Bouché $O$ et al (2007) Chemoradiation followed by surgery compared with chemoradiation alone in squamous cance of the esophagus: FFCD 9102. J Clin Oncol 25:1160-1168

2. McKenzie S, Mailey B, Artinyan A et al (2011) Improved outcomes in the management of esophageal cancer with the addition of surgical resection to chemoradiation therapy. Ann Surg Oncol 18:551-558

3. Naik KB, Liu Y, Goodman M et al (2017) Concurrent chemoradiotherapy with or without surgery for patients with resectable esophageal cancer: an analysis of the national cancer data base. Cancer 123:3476-3485

4. Stahl M, Wilke H, Lehmann N, Stuschke M (2008) Long-term results of a phase III study investigating chemoradiation with and without surgery in locally advanced squamous cell carcinoma (LASCC) of the esophagus. J Clin Oncol 26:4530-4530 